\title{
Temperature dependence of the hyperfine interaction in the cubic phase of $\mathrm{BaHfO}_{3}$
}

\author{
A. R. López Garcǐa, P. de la Presa, and A. M. Rodíguez \\ Departamento de Física, Facultad de Ciencias Exactas, Universidad Nacional de La Plata, \\ C.C. 67, 1900 La Plata, Argentina \\ (Received 30 April 1990; revised manuscript received 28 February 1991)
}

\begin{abstract}
The temperature dependence of the hyperfine interaction in the paraelectric phase of $\mathrm{BaHfO}_{3}$ in the temperature range from liquid-nitrogen temperature to $1350 \mathrm{~K}$ has been studied. The experimental results show an Abragam-Pound mechanism for the attenuation of the angular correlation. In this cubic structure the attenuation would be consistent with a relaxation mechanism that involves $\mathrm{O}$-ion hopping between vacant sites in the lattice. Using the point-charge model for the estimation of the electric field gradient produced by an $\mathrm{O}$ vacancy in the coordination sphere of the probe ion, the process is characterized by a correlation time $\tau_{c}=12 \pm 1$ ps at RT.
\end{abstract}

\section{INTRODUCTION}

The studies of perovskites by the time-differential perturbed-angular-correlations technique (TDPAC) has received the attention of several groups since the work of Glass et al. on $\mathrm{BaTiO}_{3}$ and $\mathrm{PbTiO}_{3}{ }^{1}$ These groups have been mainly concerned with the determination of hyperfine quadrupole interactions in ferroelectric (or antiferroelectric) phases and the transition to the paraelectric phase and proposed simple models to interpret the data. $^{2}$

The paraelectric phase of $\mathrm{ABO}_{3}$ has cubic structure so it had not been of interest for the application of the TDPAC technique. However, temperature-dependent perturbed anisotropies were found, for example, in $\mathrm{PbZrO}_{3}$. These attenuations could have been originated by impurities or lattice defects and the experimental data suggested dynamic effects. ${ }^{3}$ Catchen et al. ${ }^{2}$ studied the phase transition in $A \mathrm{TiO}_{3}$ with $A=\mathrm{Sr}, \mathrm{Ba}$, and $\mathrm{Pb}$ and $\mathrm{BaHfO}_{3}$. In the paraelectric phase, the attenuation factors measured were qualitatively very similar and temperature independent and they suggested that $B$-ion motion from an off-center site to another is a possible explanation, discarding either $\mathrm{O}$-ion or cation hopping between lattice sites or inhomogeneous line broadening caused by impurities, defects, and other lattice imperfections. All these experimental data confirm the existence of a relaxation process in the cubic phase of $A B \mathrm{O}_{3}$. However a mechanism responsible for the relaxation other than the $B$-ion hopping has not been proposed. To address this problem we apply the Abragam and Pound theory in the context of an O-vacancy hopping model. We present TDPAC measurements of $\mathrm{BaHfO}_{3}$ samples in the temperature range from $\mathrm{LN}$ to $1350 \mathrm{~K}$ characterized by $\mathrm{x}$-ray and thermogravimetric analysis. The interpretation of the results yields a correlation time that characterizes the vacancy hopping process.

\section{EXPERIMENTAL DETAILS}

The samples were prepared by firing at $1100^{\circ} \mathrm{C}$ for $48 \mathrm{~h}$ pellets of approximately $5-\mathrm{mm}$ diameter and less than 1- $\mathrm{mm}$ thick obtained by pressing a stoichiometric ground mixture of commercially available high purity $\mathrm{BaCO}_{3}$ and $\mathrm{HfO}_{2}$. Part of the sample was examined by $\mathrm{x}$-ray diffraction that showed the cubic pattern of $\mathrm{BaHfO}_{3}$ with a lattice constant $a=4.17 \AA .^{4}$ A second part was differential thermogravimetrically analyzed from $35^{\circ} \mathrm{C}$ to $800^{\circ} \mathrm{C}$. In this temperature range a continuous mass diminution was observed with a total loss of $6 \%$. The rest of the characterized sample was encapsulated in a sealed quartz tube at atmospheric pressure and then irradiated with a flux of $\approx 10^{13} \mathrm{~cm}^{-2} \mathrm{~s}^{-1}$ thermal neutrons to produce ${ }^{181}$ Hf TDPAC probes.

A conventional TDPAC setup with two CsF detectors and a time resolution $2 \tau \approx 740$ ps was used to accumulate coincidence count rates measured at the angles of $90^{\circ}$, $180^{\circ}$, and $270^{\circ}$. From these time spectra the perturbation factors $A_{22} G_{22}(t)$ were obtained.

Time spectra were measured at liquid-nitrogen temperature (LNT), RT, 473, 673, 873, 1073, 1273, and $1350 \mathrm{~K}$, respectively. From $473 \mathrm{~K}$ onward the temperature stability was $\pm 5 \mathrm{~K}$. After each measurement performed at 1073,1273 , and $1359 \mathrm{~K}, \mathrm{RT}$ spectra were again obtained. In Fig. 1 the anisotropy ratio as a function of time is shown for measurements at RT, $1350 \mathrm{~K}$, and RT again after the measurement at $1350 \mathrm{~K}$ plus $96 \mathrm{hs}$ at $1350 \mathrm{~K}$ in normal atmosphere.

\section{RESULTS}

All the spin precession time spectra show a similar trend: an attenuation of the anisotropy with increasing amplitude as temperature increases, slightly modulated by a static quadrupole interaction. All measurements except those at $\mathrm{LN}$ and the last at RT were performed with the same geometry so that the increase of anisotropy with temperature cannot be attributed to an experimental artifact. See Fig. 2(a)

Probes at three different sites were detected. The interaction that disappeared with temperature can by no means be fitted to the experimental time dependence of the anisotropy. It is impossible to assign it to any physical situation except that it may represent highly distorted 


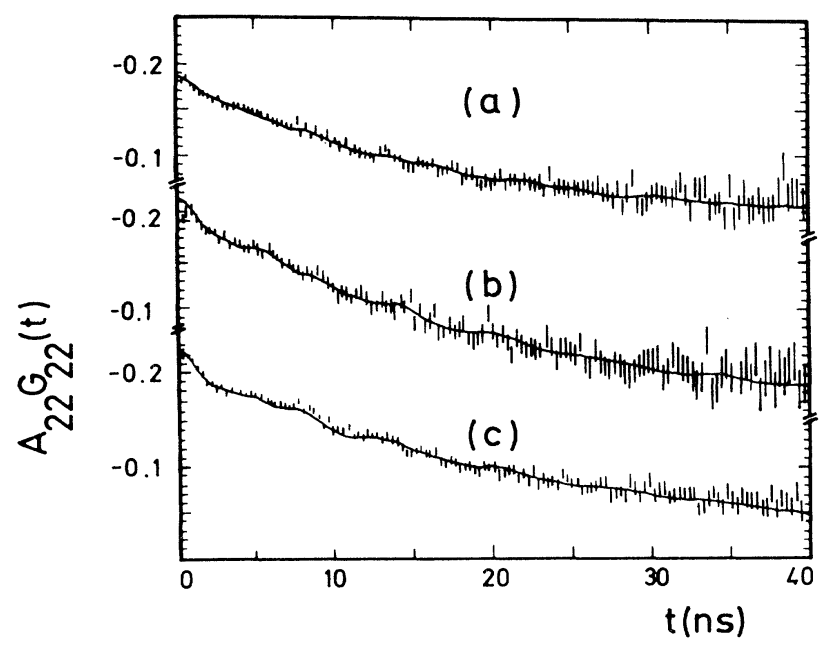

FIG. 1. TDPAC spectra of $\mathrm{BaHfO}_{3}$ at (a) RT, (b) $1350 \mathrm{~K}$, and (c) RT after the measurement at $1350 \mathrm{~K}$.

lattice sites probably correlated with radiation damage.

To extract the hyperfine parameters that characterize the remaining contributions, two models were proposed. Both have in common a static quadrupole interaction $\left(\omega_{Q 2}, \eta_{2}\right)$ slightly attenuated $\left(\delta_{2}\right)$ superimposed alternatively either to a fast time dependent perturbation or to a strongly attenuated $\left(\delta_{3}\right)$ weak quadrupole interaction $\left(\omega_{Q_{3}}=0\right)$. These attenuation models describe different physical situations, namely, a randomly fluctuating interaction due to charges independently traveling past a probe in a random manner or a cubic site with some static disorder or defects. Least-squares fits to the experimental data allowed us to compare the models and to determine the corresponding hyperfine parameters. The

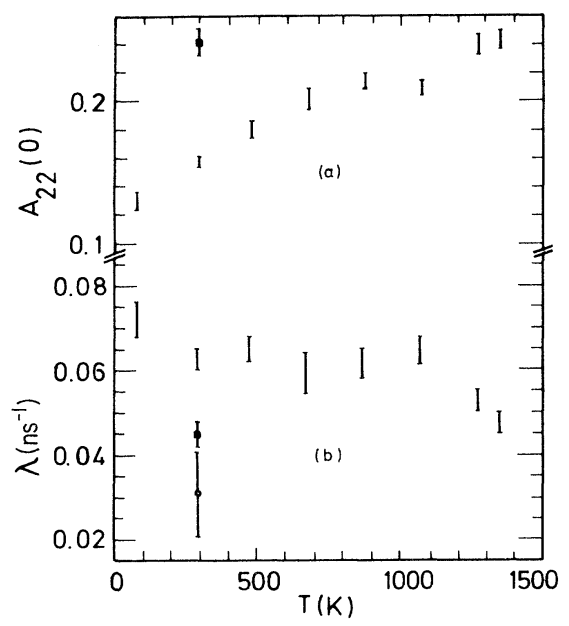

FIG. 2. (a) Temperature dependence of the zero-time anisotropy $\left[A_{22}(0)\right]$ in $\mathrm{BaHfO}_{3}$. The square represents the final RT measurement. [See (c) in Fig. 1]. (b) Temperature dependence of the relaxation parameter $\lambda_{2}$ in $\mathrm{BaHfO}_{3}$. The square represents the final RT measurement. [See (c) in Fig. 1]. last model failed to give consistent results and was discarded. Instead, the first model provided hyperfine parameters that showed consistence. Figure 2(b) shows the temperature dependence of the relaxation parameter $\lambda$.

Let us now examine the interaction at site 2 . The values obtained at RT, $f_{2}=10 \%, \omega_{Q 2}=124 \pm 10 \mathrm{Mrad} / \mathrm{s}$, $\eta_{2}=0.3 \pm 0.1, \quad \delta_{2}=0.15 \pm 0.04$ and their temperature dependence correspond to monoclinic $\mathrm{HfO}_{2}$ with some disorder or small particle size. Probably this is the reason why its presence was not detected by $\mathrm{x}$ rays. In fact the value of $\delta=0.03 \pm 0.01$ was published in Ref. 5 .

\section{DISCUSSION}

According to the x-ray analysis of $\mathrm{BaHfO}_{3}$ powder, the structure is cubic and no static hyperfine electric field gradient (EFG) is expected to occur. The thermogravimetric analysis showed a $6 \%$ mass diminution over the temperature range from 310 to $1073 \mathrm{~K}$ suggesting that gases have been lost. As in the sample we detected by TDPAC the presence of $\mathrm{HfO}_{2}(\approx 10 \%)$ probably some $\mathrm{CO}_{2}$, and barium ${ }^{6}$ remained included after source preparation and part of the mass difference can be associated to the loss of these substances. The remaining mass reduction probably could correspond to some oxygen loss. Neither the $\mathrm{HfO}_{2}$ content nor the presence of impurities or defects were, of course, detected by x-rays diffraction.

The observed attenuations of the spin precession is an unexpected fact whose origin could be associated with defects or lattice dynamics effects, or both. As we stated before, no static interaction could be fitted to the data and the best fits were obtained with a time-dependent interaction. Thus as a first conclusion some of the components of the material performed motions that are detected by TDPAC.

The temperature dependence of the relaxation parameter $\lambda_{2}$ is quite peculiar. It remains constant between $L N$ and $1073 \mathrm{~K}$ with a mean value of $\lambda_{2}^{(1)}=0.062 \pm 0.001$ $\mathrm{ns}^{-1}$. After the 1073-K measurement the value of the relaxation parameter diminished abruptly to $\lambda_{2}^{(2)}=0.045 \pm 0.003 \mathrm{~ns}^{-1}$ that also remained constant over the temperature range from RT to $1350 \mathrm{~K}$. This temperature independence of $\lambda_{2}$ was detected previously by Catchen et al. ${ }^{2}$ who obtained a value of $\lambda_{2}=0.03 \pm 0.01$ $\mathrm{ns}^{-1}$ that coincides with our $\lambda_{2}^{(2)}$.

Next, we assume that both $\lambda_{2}^{(1)}$ and $\lambda_{2}^{(2)}$ describe different time dependent defective configurations about the probes. The first one would correspond to the existence of defects whose origin and structure are not clear and are annealed out at about $1100 \mathrm{~K}$. The second one would correspond to the existence of oxygen vacancies, being usual defects in oxides. The thermogravimetric result would support this possibility.

We propose that the attenuation observed corresponds to oxygen hopping between oxygen vacant sites and assume that both heavier $\mathrm{Ba}$ and $\mathrm{Hf}(\mathrm{Ta})$ are in the corresponding cubic sites (see below). From RT to $1350 \mathrm{~K}$ we assume oxygen configurations changing fast mainly from the regular six oxygen octahedra with $\mathrm{Hf}$ sitting at the center $\left(V_{z z}^{0 V}=0\right)$ to another similar cage but with one oxy- 
gen vacancy $\left(V_{z z}^{1 V}\right)$. This change has been also assumed to be incoherent.

The experimental results have guided us to interpret the relaxation mechanism as being represented by the Abragam and Pound theory. ${ }^{7}$ This model allows us to relate $\lambda_{2}$ to $\left\langle V_{z z}^{2}\right\rangle$, the mean-square value of the EFG, and to $\tau_{c}$, the correlation time by

$\lambda_{k}=\frac{3}{80} \tau_{c}\left\langle V_{z z}^{2}\right\rangle \frac{(e Q)^{2}}{\hbar^{2}} k(k+1) \frac{4 I(I+1)-k(k+1)-1}{I^{2}(2 I-1)^{2}}$,

where $Q=2.5 \mathrm{~b}$ is the quadrupole moment and $I=\frac{5}{2}+$ the spin quantum number and parity of the nuclear intermediate state of ${ }^{181} \mathrm{Ta} \gamma-\gamma$ cascade. Hyperfine quadrupole interactions can provide information on charge distribution around nuclear probes through the hyperfine EFG. This tensor is given by

$$
V_{i j}=\frac{1}{4 \pi \epsilon_{0}} \int \rho(\mathbf{r}) \frac{3 x_{i} x_{j}-\delta_{i j} r^{2}}{r^{5}} d^{3} r
$$

where $V_{i j}$ is the $i j$ component at the origin and $\rho(\mathbf{r})$ is the total charge density at $\mathbf{r}$ and it is determined by the material. For pure ionic compounds, for example, $\mathrm{HfF}_{4},{ }^{5}$ reliable estimations can be done with a simple point charge model. In other cases, covalency or bands are also present that make the calculations of the EFG quite complicated. ${ }^{8}$ In $\mathrm{BaHfO}_{3}$ covalency has not been determined and the point charge results should be considered as a crude approximation. Taking the $\mathrm{Hf}^{4+}-\mathrm{O}^{2-}$ distance as $a / 2$ and the tabulated values of $Q$ and $\gamma_{\infty}=-61$, the point charge model gives for the EFG produced by a vacant site $V_{z z}^{(1)}=3.4 \times 10^{22} \mathrm{~V} / \mathrm{m}^{2}, \eta=0$.

Replacing these values and the mean average relaxation constants in Eq. (1) the corresponding correlation time $\tau_{c}^{1 V}=12 \pm 1 \mathrm{ps}$ is obtained. This value is faster than the $\tau_{1 / 2}=10.5 \mathrm{~ns}$ of $\mathrm{Ta} \frac{5}{2}+$ excited state and also $\tau_{c}\left(\left\langle\omega^{2}\right\rangle\right)^{1 / 2}<1$, conditions that must be satisfied.

In studying the crystal structure of $\mathrm{BaTiO}_{3}$ at temperatures between the Curie temperature and about $453 \mathrm{~K}$, Itoh et al., ${ }^{9}$ observed that $\mathrm{O}, \mathrm{Ti}$, and $\mathrm{Ba}$ atoms are displaced from their "cubic" sites in a disordered way and at temperatures higher than about $453 \mathrm{~K}$ the $\mathrm{Ti}$ atoms lie at the center of the cube. The first facts, namely, the miscentering of atoms could tempt us to interpret the observed relaxation as originated by the positional disorder. Using the point charge model, this contribution to the EFG is found to be $2-3$ orders of magnitude weaker than that produced by an oxygen vacancy. Thus the above mechanism could not describe the observed facts. A second reason is that the temperature range of our experiment is far from the transition temperature.

The temperature dependence of $\lambda_{2}$ is peculiar. It seems to depend on probe-defect structures, and could correspond to either interstitial-vacancy complexes or to different vacancy complexes. The first system should be more unstable with temperature than the second one. To analyze the last possibility let us compare our results with those in other systems like $\mathrm{ZrO}_{2}-\mathrm{Y}_{2} \mathrm{O}_{3}$ and $\mathrm{ZrO}_{2}-\mathrm{CaO} .^{10}$ In these cases, the $O$ vacancy concentration is in the order of tens of percents and causes a strong temperature dependence of $\lambda$. For $\mathrm{BaHfO}_{3}$, the $\mathrm{O}$ vacancy concentration is expected to be no more than a few parts percent. The vast difference in the $\lambda$ temperature dependence of $\mathrm{BaHfO}_{3}$ as compared to $\mathrm{ZrO}_{2}-\mathrm{Y}_{2} \mathrm{O}_{3}$ may be produced by the diversity in their $\mathrm{O}$ vacancy concentrations.

\section{CONCLUSIONS}

Using the TDPAC technique we have studied the temperature dependence of the hyperfine interaction in $\mathrm{BaHfO}_{3}$. This simple perovskite shows cubic structure in the temperature range from $\mathrm{LN}$ to $1350 \mathrm{~K}$. The perturbation of the anisotropy observed has been associated with dynamical effects originated by $\mathrm{O}$-ion hopping between vacant oxygen sites. By means of the Abragam and Pound model and the point charge model for the EFG at the sites of the probe nuclei we have deduced a hopping time of $12 \pm 1$ ps at RT.

\section{ACKNOWLEDGMENTS}

We thank Dr. M. Benyacar for the X-ray analysis and interesting comments, Dr. M. Weissmann for fruitful discussion, and Lic. Griselda Polla for the DTG analysis. two of us (A.L.G.) and (A.M.R.) acknowledge CONICET for partial financial support.
${ }^{1}$ J. C. Glass and J. K. Kliwer, Nucl. Phys. A115, 234 (1968); M. R. Hass and J. C. Glass, Phys. Rev. B 4, 147 (1971).

${ }^{2}$ G. L. Catchen, S. J. Wukitch, E. Saylor, W. Huebner, and M. Blaszkiewicz, Ferroelectrics (to be published).

${ }^{3}$ G. Teisseron and A. Baudry, Phys. Rev. B 11, 4518 (1970).

${ }^{4}$ M. A. Berchenko and I. A. Belyaer, Russ. J. Inorg. Chem. 15, 1034 (1970).

${ }^{5}$ M. C. Caracoche, J. A. Martinez, P. C. Rivas, and A. R. López García, Hyperfine Interact. 23, 226 (1985).

${ }^{6}$ A. E. Panich, S. M. Maksimov, O. I. Prokopalo, and E. G.
Fesenko, Inorg. Mater. 15, 696 (1979).

${ }^{7}$ A. Abragam and R. V. Pound, Phys. Rev. 92, 943 (1953).

${ }^{8}$ A. R. López-García, Magn. Reson. Rev. 15, 119 (1990).

${ }^{9}$ K. Itoh, L. Z. Zeng, E. Nakamura, and N. Mishima, Ferroelectrics 63, 29 (1985).

${ }^{10}$ A. Baudry, P. Boyer, and A. L. de Oliveira, Phys. Lett. 79A, 345 (1980); J. Phys. Chem. Solids 43, 871 (1982); J. A. Gardner, H. Jaeger, H. T. Su, W. H. Warnes, and J. C. Haygarth, Physica A150, 233 (1988). 